\title{
General Unificatory Theories in Community Ecology
}

\author{
Christopher Hunter Lean
}

University of Sydney

\begin{abstract}
The question of whether there are laws of nature in ecology has developed substantially in the last 20 years. Many have attempted to rehabilitate ecology's lawlike status through establishing that ecology possesses laws that robustly appear across many different ecological systems. I argue that there is still something missing, which explains why so many have been skeptical of ecology's lawlike status. Community ecology has struggled to establish what I call a General Unificatory Theory (GUT). The lack of a GUT causes problems for explanation as there are no guidelines for how to integrate the lower-level mathematical and causal models into a larger theory of how ecological assemblages are formed. I turn to a promising modern attempt to provide a unified higher-level explanation in ecology, presented by ecologist Mark Vellend, and advocate for philosophical engagement with its prospects for aiding ecological explanation.
\end{abstract}

[T] he case for laws in ecology is generally thought to be weaker, since ecology lacks a grand, widely-accepted, explanatory theory such as Darwinian evolution. -Colyvan and Ginzburg 2003, 651

\section{INTRODUCTION}

The question of whether there are laws of nature in ecology has developed substantially in the last 20 years (Colyvan and Ginzburg 2003; Ginzburg and Colyvan 2004; Lange 2005; Linquist 2015). There is a new focus on the robust and resilient generalizations that ecological science produces (Linquist et al. 2016). This is a positive development, opening new avenues for identifying causal relations that can be implemented in 
practical responses to the global environmental crisis. Despite these developments I contend that there is more to say on questions of whether there are ecological laws of nature. The flood of skepticism toward laws of nature in the 1990s was built around the failure of general theories that applied widely to community ecology (Lawton 1999; Peters 1991; Shrader-Frechette and McCoy 1993). It had become apparent that many of the top-down general theories of ecological composition rarely applied to actual ecological systems, which fueled skepticism toward ecology's status as a science. This skepticism was coupled with a strong belief that local explanatory models and predictions were insightful. Nonetheless, the lack of general theory, I argue, still causes problems for explanation in ecology as there are no guidelines for how to integrate the local mathematical and causal models into a larger theory about the way ecological assemblages are formed.

This concern could be described through the language of Philip Kitcher's unificationism (Kitcher 1981). Successful scientific theories, according to unificationists, have an argument pattern built from a schematic sentence, which can derive descriptions of many distinct empirical phenomena. The satisfaction of the unificationist urge to explain a large set of phenomena, in one type of schema, is part of the worry I am describing but not quite it. Unificationism is often coupled with a winner-take-all problem in which the most unificatory theory is the most explanatory (Woodward 2017). This I reject. The major developments in the philosophical literature on scientific 
explanation over the last 20 years have been based around local explanatory models, be these interventionist causation, mechanisms, or models (Batterman and Rice 2014; Craver 2007; Weisberg 2013; Woodward 2005). All of these do not aim solely to unify a large number of phenomena but instead focus on, and trade between, other explanatory virtues including accuracy and precision. I contend that it is critical for a science to have both higher-level explanations, that are unificatory and general, and lower-level explanations, which are precise as they contain more detail in their description of the phenomena and predictive power.

Ecology lacks explanatory integration in the sense that there is no general and unificatory theory, a General Unificatory Theory (GUT). A GUT is general in that it can apply to many distinct actual systems but also unificatory in that it can apply to much of the sciences target explanandum, often in an imprecise way. These broad and slightly imprecise theories are critical as they provide a structure into which we can place lowerlevel less unificatory theories. Lower-level theories explain details of the phenomena the GUT does not but remains silent on the larger system explained by the GUT. What I contend is that without a GUT, the science is impeded because the lower-level piecemeal theories are left as free-floating unrelated inferences, and there needs to be a higher-level comprehensive theory to guide how these theories relate.

Community ecology's many, well-supported, but piecemeal explanatory models have been unable to be related through the framework provided by a GUT. The 
relationship between 'local' model explanation and 'global' theories is described by Andrew Wayne as explanatory integration (Wayne 2018). Global theories have explanatory power independent from the local models as they unify phenomena and provide a schematic to derive predictions from initial conditions. These predictions are often highly idealized and difficult to implement to actual systems but provide a broad picture of the way different empirical phenomena relate within a science. Local theories are much more precise and implementable. They can describe actual instances of natural phenomena in detailed and predictively accurate ways.

Within Wayne's terminology an explanation is either global or local. This distinction, however, does not fully capture the dimensionality of how laws apply, which is why I have altered my terminology to less elegant 'lower-level' and 'GUT'. Laws can be general but not unificatory. General explanations apply to many different systems, despite changing local background conditions. Unificatory explanation, as I am using it, applies to the entirety of the sciences target explanadum; it acts to unify the different explanations of different parts of the target system. Think of the way natural selection provides explanatory power to so much of biological phenomena. In community ecology, the entire target system is the local ecological community, so our explanadum is the compositional identity of that community. Why do the species that exist in that community appear there and what causes their abundance? A unifying and general explanation is one that is explanatory for the entirety of local ecological communities, 
for many communities. It should provide some information about all the populations that appear within these local communities even if highly idealized. Given this conception of explanation, explanations for some feature, in many communities, is not a unifying explanation for community ecology.

If one is to agree with Wayne (2018), there is an important project of mapping the relationship between local theories, what I am calling lower-level theories, and the mutual supporting and organizing global theories (GUTs). This is done through a series of abstractions, idealizations, and counterfactual reasoning (p. 352). I will not address the details of this 'mapping' relationships project but wish to look at the issue of not having any suitable higher-level theory to organize and relate lower-level explanations. In ecology, we have many successful local theories, but we lack an organizing global theory of ecological composition - the distribution, identity, and abundance of species in a local ecological community—and this ultimately undermines ecology's lawlike status.

I read the history of skepticism toward laws in ecology through this prism. The fear of ecology's lawlessness was always focused around the lack of a unifying global theory that could explain ecological composition. In the following section, I turn to modern replies to the laws debate and show how they provide accurate lower-level explanations. Then in section 3, I attempt to characterize why lower-level ecological explanations are disunified and why this hinders explanation. Finally, I turn to a modern attempt to 
provide a unified higher-level explanation in ecology and advocate for philosophical engagement in its prospects for aiding ecological explanation.

\section{INVARIANCE IN ECOLOGICAL EXPLANATION}

The laws of nature debates in ecology have largely focused on the role of invariance in explanations. A common interpretation of laws of nature has them exist as invariant and universal generalizations (Smart 1959; 1963). Anxiety about the existence of laws of nature has largely subsided through relaxing of the conditions under which a scientific generalization qualifies as a law, permitting laws which are not perfectly universal or invariant. Accordingly, ecology has found itself possessing laws through these relaxed standards (Cooper 1998; Colyvan and Ginzburg 2003; Lange 2005; Linquist et al. 2016). These relaxed standards, of what counts as a law, have been perceived as a reply to skeptics of general laws in ecology (e.g., Lawton 1999; Peters 1991; Shrader-Frechette and McCoy 1990, 1993). I pay attention to the work of Stefan Linquist (2015; Linquist et al. 2016), who has developed a comprehensive recent assessment of the prospect of laws in ecology.

Through the 1980s and 1990s there was a growing disillusionment with ecology's ability to be a successful science. Some targeted ecology's inability to make accurate predictions, leading them to describe it as a 'soft science' akin to sociology rather than the rest of biology (Peters 1991). Ecology's lawlessness appeared particularly acute in 
community ecology, where many practicing ecologists feared there were no lawlike generalizations (Brown 1995; Ricklefs 1987; Roughgarden 1989). Two exemplars of the skepticism toward ecological laws were John Lawton (1999), who argued against the existence of 'general laws' in ecology, and Kirsten Shrader-Frechette and Earl McCoy (1993), who argued that 'top-down' explanation, using general theories, fails in ecology and instead explanation should be built 'bottom up' from case studies. Both are skeptical of what I would consider GUTs of ecological communities, which aim to explain widely the demographic features of an ecological community in an area. Theories like niche theory, assemble rules, or the diversity stability hypothesis all aimed to describe broad demographic trends in a community. It is to these theories that skepticism toward laws was directed rather than local mathematical and causal laws, which both at times appear to affirm. ${ }^{1}$

In the years since there has been a defense of laws, stating that there are widely applicable laws in ecology even if they are not universal. Some interpret critics like John Lawton (1999) as requiring ecological laws have universal and invariant generalizations (e.g., Roughgarden 2009). Given this perspective no ecological law would qualify, and we should reasonably reject this high standard for law-hood. The responses developed

\footnotetext{
${ }^{1}$ For example: Shrader-Frechette and McCoy state "despite the problems with general ecological theory, there are numerous lower-level theories in ecology that provide reliable predictions" $(1993,120)$. Lawton states "contingency is manageable at a relatively simple level of ecological organisation (for example the population dynamics of single and small numbers of species)" $(1999,177)$.
} 
to skeptics of laws in ecology have a firm basis in the philosophical literature. There is a long history, in philosophy of science, of arguing that laws of nature can be contingent, and exception ridden (Cartwright 1983). Accepting ecology as full of contingent ceteris paribis ridden laws was argued early. Greg Cooper (1998) held that in ecology, just as in the rest of biology, universality is not a reasonable standard to hold laws to. Instead law status should be derived from the range of different contexts the law holds over; the more contexts, the more law-like it is. This move was in line with several suggestions aiming to weaken the conditions under which a natural pattern qualifies as a law (Mitchell 2000; Skyrms 1980; Woodward 1997). Contingency was astutely treated as a quantity that came in degrees, rather than a binary presence. Degrees of contingency, the extent to which a phenomenon is robust to changes in background conditions, is of the utmost interest to science. This intellectual shift provided opportunities for ecology's contingent generalities to become lawful.

It is within this space, of identifying degrees of invariance, that we find important replies to the earlier criticisms of the possibility of ecological laws. Linquist (2015) argues that ecology is rich with causal relations, which are invariant across a range of background conditions, and applies the conceptual apparatus of James Woodward (2005) to defend the existence of laws in ecology. Degrees of invariance can be explicated utilizing the work on causation and laws Woodward developed (1997; 2005). Causation is understood as a relationship between two variables $X$ and $Y$, if there is a 
systematic intervention on variable $X$ to change its value and this results in a change in the value of variable $Y$, then $Y$ causally depends on $X$. These causal relations are always within a context of background conditions (B). Water boils at $100^{\circ} \mathrm{C}$ in many different conditions, but in some conditions it doesn't. For example, variation in the degree of air pressure or percentage of salt present can change the value of the target variable. A causal relation can be 'stable' or 'contingent' given the degree to which the causal dependency is found across a range of background conditions.

A similar defense of the existence of contingent laws can be found in Mark Colyvan and Lev Ginzburg, who, among others, argued there are imperfectly instantiated mathematical laws (Colyvan and Ginzburg 2003; Ginzburg and Colyvan 2004; Turchin 2001). ${ }^{2}$ Lawton also accepts these as laws $(1999,177)$. Prime candidates of mathematical laws of ecology being the logistical growth of populations or allometries, like the Kleiber allometry, which states that an animal's basal metabolic rate is $3 / 4$ of its body mass.

These laws are mathematical and statistical generalizations that apply widely in nature. They are subject to contingency; changes in initial conditions can alter the presence of such patterns. For example, population growth is not logistical when there are disturbing factors (see Kingsland 1985 on the historical debates on population growth).

\footnotetext{
${ }^{2}$ A reviewer questions if such laws are laws of mathematics rather than laws of ecology. I do not make such divisions. The gas laws are laws of gasses as much as they are laws of mathematics. Causal laws are laws of the target variables as much as they are instantiations of the metaphysics of causation. Following Lange (2005), what makes a law a law of a particular science is the explanandum under consideration.
} 
Ecological laws appear to apply across multiple different system types, such as different habitats or temperatures. A law can be robust in the face of setting a background variable to different values or variation in the variable itself. The ability of a generalization to survive changes in the background variables is described by Linquist et al. 2016 as resilience and they describe three different categories of variation in background variables. They describe resilience in the face of variation in the species the generalization holds over (taxonomically resilient), variation in habitats (habitat resilient), and variation in the scale (spatially resilient) ${ }^{3}$. Then using meta-analyses of multiple ecological studies, they identify laws that have such resilient generalizations. They identified generalizations in community, ecosystem, and population ecology. These included: habitat fragmentation negatively affects pollination, herbivore removal increases primary producer biomass, and invasive species increase pools of stored nitrogen.

These generalizations are very different to the generalization of niche theory, assemble rules, or the stability-diversity hypothesis. They are significantly more constrained in their scope. The target variables are not area and species diversity, or

\footnotetext{
${ }^{3}$ I have found this description of resilience rather counterintuitive. For example, take the following generalization: warmer temperature increases species $\mathrm{X}$ predation success. The target variables are temperature and the predation success of a species. Both these variables take a range of values. Linquist et al.'s 2016 notion of resilience would have this resilient if it was generalizable over many species, but this is not a background condition, it is a change in the target variable. Resilience, as I would consider it, would involve varying not the temperature or species, the target variable, but other background conditions like the habitat type this taxon was present in to see whether the relationship held. But my focus here is on other issues rather than what variables are background conditions in causal explanation.
} 
total resource consumption, which can then be used to describe the entire community species count or identity. Instead these variables are aimed at units of investigation like pollinators or invasive species, parts of the ecological community. These are crucial explanandum but are not the sort of variables act to describe broad features of a local community. These replies are correct in establishing there are resilient laws. Precise lower-level explanatory models in ecology are well supported and quite general in that they apply to many systems under a range of background conditions. But these resilient lower-level laws do not establish the presence of top-down GUTs in community ecology. Many of the replies to ecology law skeptics have involved showing the range of conditions a generalization is maintained over, but this has not affirmed a role for general and unificatory theories about what determines the distribution and abundance of organisms in a community. Some may consider this an acceptable position for ecological science. We could have many different robust lower-level theories and models without a larger theory of ecological composition. In the next two sections, I turn to characterizing the problems with solely having lower-level generalizations and elaborate a promising recent attempt in the ecological literature to provide a GUT of ecological communities.

\section{GUT-LESS SCIENCE ${ }^{4}$}

\footnotetext{
${ }^{4}$ Please do not interpret this as inflammatory. There was an opportunity for a pun, so I took it.
} 
Local ecological communities have robust local causal structures that generalize across multiple instances. The contents of these 'laws' are, however, extremely disjunctive. They describe different kinds, patterns, and processes, rarely in ways that easily relate to the contents of other laws. The purpose of a general theory in a science is to structure the predictions of lower-level laws so that they can ultimately relate their predictions to each other. The robust generalizations need to speak to the larger scientific field they belong to. Without general theories, these lower-level explanations often only provide information about their local explanandum, and they struggle to inform explanandum which fall outside the local model. A general scientific theory informs how a local model relates to other explanadum across their science.

The disunity of lower-level laws can be observed from their drastically different variables (Vellend 2016). Consider two of the laws of nature identified by Linquist et al. (2016) for community ecology, "The removal of herbivores increases the biomass of primary producers" and "The impact of grazers on prey biomass decreases in proportion to species richness of prey communities" (Table 2, p. 11). The variables in just these two rules include a count of herbivores, a quantity biomass of primary producers, a quantity of prey biomass, a count of prey species types. Both biomass and counts of individuals are used. Generally, this is because defining individual organisms for many species, particularly plants, is extremely difficult so ecologists tend to use biomass for organic 
types that they cannot easily individuate. As such, sets of organic types are bifurcated into continuous or discrete measures. Distinguishing organic types is extremely difficult in ecology. We have herbivores, prey, grazers, primary producers, and species. These different taxonomies for organic types include classical biological taxonomy, species, but also several different ways to carve up food chains and functional actions in a community.

While plenty of sciences involve many unique variables, for example chemistry involves a large number of individual chemicals, what differs in ecology is the lack of theory guiding the description of these variables. Chemical kinds have an explanatory framework by which they are divided, their atomic number. This acts to structure the inferences made about these different variables and combinations made from them. There is a GUT that structures the variables and provides a way to relate the variables. Ecological variables are built up from many unique local models and frameworks, which impede this ability to relate explanations in one lower-level theory to another. But how do GUT act to organize the inferences made through lower-level theories?

Prominent general theories in other sciences are evolutionary theory, the standard model of particle physics, or the general theory of relativity (Wayne 2018). All these theories act to structure the lower-level models within their respective sciences. Wayne (2018) uses the example of R. A. Fisher's sex ratio model, which identifies an equilibrium dynamic resulting in a 1:1 sex ratio. This local model shows the sex ratio, like many 
biological phenomena, is subject to stabilizing selection. Stabilizing selection can be taken as evidence for very different conclusions. The sex ratio model can be taken as evidence for creationism; many different changes to mating strategies will gravitate toward this stable ratio. As such, there is a preordained structure in nature, 1:1 ratio sexually reproducing species, which is resistant to changes. The impact of this model on the science of biology is much different when it is embedded in the theory of evolution. The meaning of the local model, the sex ratio model, to the scientific field, biology, is structured by the general theory, evolutionary theory.

Sex ratios will be robust across multiple different biological systems, it is a resilient feature across biology. Without the more general unificatory model of natural selection there is no indication of why this generalization holds in some cases and not others. ${ }^{5}$ Exceptions exist to sex ratios and it is the larger theory of natural selection that provides us with the framework to understand why. When a lower-level model is embedded in a network of more general models we are given a guide to why the law applies. It is not enough to simply have a theory be robust in local instances, we want to know why it is robust under the background conditions it is. This is what general unification theories provide, guidance on when a model should apply to a target system.

\footnotetext{
${ }^{5}$ Thanks to Stefan Linquist for a helpful discussion on this point.
} 
Applying this to the case at hand, consider two laws as proposed by philosophers of ecology, Kleiber allometries, and "the impact of grazers on prey biomass decreases in proportion to species richness of prey communities" (Colyvan and Ginzburg 2003; Linquist et al. 2016). Kleiber allometries are found widely in nature but it remains extremely unclear as to why they hold. There is an explanatory gap, which if they were placed in a larger theory we would be in a much better epistemic position to answer. As of yet, they stand as a generalization without clear reason as to why they are general. This is similar to Kepler's laws in astrophysics; they are extremely robust and widely found but it was unclear as to why they apply without a more unificatory law like Newtonian Gravity. ${ }^{6}$ Equally, "the impact of grazers on prey biomass decreases in proportion to species richness of prey communities" involves very specific sets of populations; why does this theory only apply to grazers and not carnivores or insectivores and why is the law-like relation quarantined to this set of causal variables and why not form a law-like relationship with the larger set of species that interact in this community? While we can hypothesize why this is the case, a more unificatory theory would provide clear guidance as to why rather than requiring much secondary hypothesizing.

${ }^{6}$ Thanks to Mark Colyvan for bringing up this analogy. 
The exemplar of a GUT in community ecology was niche theory (Elton 1927; Hutchinson 1957; Macarthur 1958; Macarthur and Levins 1967). Niche theory acted to integrate a whole large set of observations and generalizations into a single framework allowing for their comparison. Describing an organism's niche involved providing a profile of the resources it used. In doing this, ecologists could identify resource overlaps and resources over which populations competed. Resource consumption could then be placed within a food web to identify which populations consumed each other and influenced the larger resource cycle. Ultimately, this connected both the biotic dynamics of food web and competition with abiotic resource cycling, providing a comprehensive picture of ecological communities. The focus on competition in niche theory allowed for ecological dynamics to be continuous with evolutionary theory, as competition is considered a major driver of adaptive variation. Niche theory was an elegant global theory, which comprehensively explained ecological phenomena at the local community level and the role of community ecology in the biological sciences. Due to its comprehensiveness, the lower-level models of ecology could be incorporated into the niche theory framework. With niche theory losing its centrality to ecology through the 1980s and 1990s there was a lack of organizing grand theories in ecology (but see Chase and Leibold 2003 for a defense of niche theory).

The failure of the GUTs proposed in ecology, particularly niche theory, had and has a real impact on ecology. Global theories provide an explanatory blueprint for how the 
lower-level variables are related. Without this explanatory structure we are faced with a series of problems, which I now elaborate. First, there is the Tower of Babe/problem. There is an immense set of redundant, and worse, partially redundant variables described in scientific laws. The partial co-descriptive nature of these variables causes problems for when we try to represent their causal influence on each other, this I describe as the (Partial) Partitioning of Causes problem. The third issue I want to raise is the possibility there is no way we can translate the tangle of variables to each other, that some variables in ecology are Incommensurable. All these issues result from having no general framework from which to relate the different variables in ecology.

\subsection{TOWER OF BABEL}

The Tower of Babel problem is well known by ecologists (e.g., Grimm and Wissel 1997; Herrando-Pérez et al. 2012). It is where the terminology used to describe ecological phenomena is so inflated and inconsistent it is difficult to parse the relationships of the concepts and scientific variables used in ecological research. This may not seem significant to some philosophers, as they might conclude that there is still some ideal way for these terms to be related if we are given time to find it, but it is immensely impactful to practicing scientists (Trombley and Cottenie 2019). I explain why terminological convergence and variable consistency aids scientific progress. 
Consistency is often a forced byproduct of having to relate each lower-level theory to the general theory that subsumes them.

For each of the important variables in ecology we find a proliferation of similarly referring terms. Terms such as 'ecological community', 'ecological function', 'invasive species', 'biodiversity', and 'niche' all have a wide range of interpretations through the science. In an extended consideration of the concept density-dependency, HerrandoPérez et al. (2012) identify several different manners in which the lexicon is counterproductively inflated. ${ }^{7}$ Term inflation can include synonymy, where two words refer to the same thing. For example, the observation that increasing population size results in the slowing of population growth has been referred to as 'centripetality', 'compensation', and 'disoperation'. Such a problem can be addressed by convention, if we can bring all parties into agreement. A more difficult issue is when terms differ slightly but not substantially. This can be due to differing interpretations of the theoretical justification of the terms meaning or the revision of the term so that it includes more phenomena the researcher considers explanatorily important. This results in multiple terms that partially co-refer. Partial co-reference yields problems in partitioning causal influence, as discussed below, but beyond that it leads to considerable confusion about what phenomena has been explained. A general theory,

\footnotetext{
${ }^{7}$ The ideas in this paper are expanded and clarified in the following blog post by the coauthor Corey Bradshaw. https://conservationbytes.com/2012/09/17/ecology-tower-babel/ (accessed February 8, 2019).
} 
which each of these terms need to be related, allows for a common language that new variables must be coherent with and allows for consistent meaning across a science.

Inconsistent and difficult to parse terminology holds back the education of the next generation of ecologists. When students and early career researcher try to understand concepts, concepts that experts reasonably disagree on, the lack of clarity puts a barrier to understanding the difficult theoretical terms that populate the science. This does not just impede the speed at which experts can be trained; some within the field may avoid research which deploy such byzantine terminology. This in the long term will influence the scope of ecological research and can drive the turn to studying individual populations, or narrow research domains, rather than broader more theoretical hypotheses. This is a different type of problem than what are usually considered by philosophers. The lack of unifying explanations is creating a practical problem: it prevents scientists from identifying a framework that can aid in organizing the terminology. Instead convergence is case by case and requires difficult negotiations between relevant scientific parties as they lack a common worldview. The science through a lack of an epistemic resource, a unifying framework for their target phenomena, is practically impeded.

\section{2 (PARTIAL) PARTITIONING OF CAUSES}


The differing local theoretical frameworks can result in many sets of variables, which extensionally overlap or partially overlap. When we want to relate these local laws, or models, we need to identify how the variables in each law causally or constitutionally relate to each other. The process of identifying these relations can be extremely difficult. Decisions must be made about what general framework is used to relate all these differing lower-level theories. A general theory of ecological communities would provide a series of variables which lower-level theories would relate to creating a common causal and constitutional framework. I believe that there is a real problem with just building a science from the ground up without any consistency in the variables used.

The importance of variable consistency can be seen when we incorporate ceteris paribus conditions or integrate different laws on a single target phenomenon. Colyvan and Ginzburg (2003) describe the Galilean law of freefall, in which large bodies all fall at constant acceleration irrespective of their mass. This is a well-established law of physics, but it has well-known exceptions. Snowflakes have radically different rates of acceleration than hailstones. This is due to the ceteris paribus condition of friction, which is derived from Newtonian mechanics. When these two laws are integrated, to explain the difference between the phenomena of snowflakes and hailstones falling, we need to identify their differential effect on the target phenomena, the acceleration of these bodies. Both laws can have their causal influence partitioned in their effect on 
single target variable. This is required for integrating causal relations derived from separate laws.

The partial co-reference of variables to the same system results in problems when we attempt to integrate different laws. Local laws, which display causal relationships between variables, will eventually need to be related to each other to gain a comprehensive picture of how ecological systems are arranged and maintained. We need to identify whether the local laws just redescribe the same phenomena (and the relationship between variables) in different local laws. When different laws describe variables that partially refer to the same referent we will have a problem when we amalgamate the causal information contained in these laws. For example, Law X describes the conditional relationship A causes B, Law $Y$ describes the relationship C causes $B^{*}$, where $B$ and $B^{*}$ partially refer to each other. When we want to unify the science, we need to decide what the dependent variable is in the natural system we want to explain, $B$ or $B^{*}$. When settling on either variable, or a third compromise variable, there will be difficulty in identifying how much $A$ and $C$ influence the dependent variable due to the partial reference. It may require a whole new set of research to identify the relationship between these variables, slowing down progress and impeding our ability to infer the impact of lower-level laws on each other. These impacts could act as background conditions for other laws, which explain why certain 
laws have the general domain they do. As such, having the ability to identify the network of causal interactions between local laws is crucial for scientific understanding.

\subsection{INCOMMENSURABILITY}

The distinct carving of ecological variables might be untroubling, in the ideal, if these variables could be translated from one variable set to another. This would involve finding systematic correlated relationships between the values in one variable description and another. Translation between variable sets is, however, not possible if variable sets are incommensurable. In such cases there is no systematic way to translate the variables found in one theoretic description to another. For example, there is a clear relationship between the measures of an imperial measurement 12 -inch ruler and a metric $30-\mathrm{cm}$ ruler but there is not a systematic relationship between the values of an imperial measurement 12 -inch ruler and the Schmit sting pain index. The incommensurability between the Schmit sting pain index and a classroom ruler is acceptable because both apply to very different representational aims; one is to measure distance, the other the pain inflicted by insect (Hymenoptera) stings. Community ecology suffers from a lack of commensurability internal to its own field, to features with similar representational goals. This causes problems for integrating lowerlevel laws in ecology as the variables in one model, or local law may not be able to be translated into the other. 
The problems I am describing in community ecology have been previously ascribed to a narrower domain of microbial ecology by Inkpen et al. (2017). They focus on the problem of functional measures in microbial ecology and argue there is no systematic way to distinguish functional types in an ecological setting. This fear about functional measures has been voiced in macroecology with Mlambo $(2014,781)$ stating loose definitions of function result "in an unfortunate view of functional diversity as a combination of any available morphological, physiological and life-history traits." This ultimately makes function incommensurable with taxonomic variables (often measured through phylogenetic diversity) as what counts as a function is highly disjunctive. This coheres with a growing body of literature, which show a lack of a clear relationship between these measures (Devictor et al. 2010; Mazel et al. 2018). There can be correlations, but these will be partial and interestingly asymmetrical; taxonomy may predict function while function is inaccurate at predicting taxonomy. I would, however, restrict this worry to functional measures that describe morphological or physiological traits rather than function in the sense of cycling a type of resource. Resource cycling is a more multiply realizable trait and can appear in many more varied contexts, and this allows it to avoid incommensurability with other traits.

Incommensurability between phylogeny and morphological function matters because both the measures have similar representational aims, describing the diversity of populations in an ecological setting. Functional measures are generally local to 
limited sets of species, rather than the majority of life, and functional measures are highly disjunctive, sharing little in common, so there can be no consistent relationship between function and taxonomy. The disjunctive nature of function through life explains the incommensurability between it and phylogeny. Some critics have argued against using phylogenetic measures of diversity because they do not accurately correlate with function, and function connects better to ecosystem service (Winter, Devictor, and Schweiger 2013). Other argue the wider representational scope of phylogenetic measure makes them preferable as a general measure of diversity (Lean 2017). Both sides aim for a convergence on key measures of diversity to integrate the ecological research being produced and aid conservation practice. Given the lack of consistent translation between function and taxonomy, be it species taxonomy or phylogenetic relations, these measures are incommensurable along the entire set values these variable sets take. There cannot be a single set of bridging laws that explains the relationship between these two measures.

Incommensurability can result from having variables uniquely individuated by many different scientists and different conceptual schemas. Invasive species are alleged to be examples of this. The category is famously underdetermined with no consistent usage; what counts as an invasive species from one study to another can differ widely (Rejmánek et al. 2002). There seems to be no clear biological properties underlying 'invasive species' which would allow for a translation of invasive species to some sort of 
functional biological variable (Sagoff 2005). Incommensurability, as a result, pervades community ecology and extrapolating from one study design to another varies from extremely difficult to near impossible.

This all comes to a head in attempting to explain an ecological community's composition, what explains the current distribution and abundance of populations. To explain a community, we need to partition up the unique causal influences that affect the community into discrete causes that can account for all the variation. We need to know why certain resilient laws appear to be robust over some background conditions and not others. A cacophony of non-discretely partitioned and incommensurable variables cannot be adequate for explanation. For a complete theory we need a general account of ecological arrangement, in which the variables can be partitioned so the lower-level laws can be related. This would allow for a consistent science of ecological communities, which can allow for studies across the science to be relatable.

\section{A RECENT PROPOSAL}

General Unificatory Theories never left ecology after the panic over whether ecology possessed laws of nature. A recent development is Mark Vellend's description of community ecology being governed by four higher-level processes in his book The Theory of Ecological Communities (2016). He defends the need for a general and coherent picture of ecological communities. This theory uses a general description of 
ecological community phenomena that is consistent in its variables, economical, and largely complete. This is not to say that it is correct, there is a way to go to establish that, and I think there remains significant problems with Vellend's proposal. However, this theory is a major development and an exemplar of how a general theory organizes the local models and causal inferences, with disparate variables, into a single framework. This aim, to unify lower-level theories into a single general theory, is also explicitly Vellend's aim.

In a series of papers and later a book Vellend argues that community ecology can be viewed as analogous to population genetics (Vellend 2010; 2016; Vellend and Orrock 2009). Both sciences aim to explain patterns of distribution and abundance. In population genetics it is the arrangement of genetic characters in a population, while in ecology it is the arrangement of organism types in a community. Population genetics explains the arrangement of genetic characters in a population through the higher-level processes of selection within species, drift, gene flow, and mutation. These processes are not described by single causal structures or mathematical pattern. They are explained through many lower-level causal relations and represented by families of mathematic models. But they are unified at the higher level through general processes that describe how varietals move in a population.

The theory of ecological communities posits ecological communities are arranged by the following forces: selection among species, drift, dispersal, and speciation. These 
are analogues of the four central processes in population genetics-selection within species, drift, gene flow, and mutation. Whereas the target variables of population genetics are the change in allele types, the target variables of community ecology are the change in species types. The selection between species has been well studied and much of what was historically described as 'traditional community ecology' by authors including Lawton focused nearly entirely on selective forces between species types (Brown 1995; Lawton 1999). Speciation involves the production of new species varieties, just as mutation produces new varietals in genetics. Macroecologists have emphasized the influence of speciation of local community organization (Brown 1995; Ricklefs 1987). Drift incorporates the stochastic aspect of birth, death, and mating; as such, this theory includes probabilistic influences on the arrangement of species. The final process is dispersal, which accounts for how species move across a landscape, which is heavily influenced by metacommunity structure and the scale of the community.

This framework is aimed to be comprehensive, with Vellend stating "despite the overwhelmingly large number of mechanisms thought to underpin patterns in ecological communities, all such mechanisms involve only four distinct kinds of processes: selection, drift, speciation, and dispersal"(Vellend 2010, 183). Through maintaining a single set of target variables, the distribution of organisms as typed through species, and having an exclusive set of forces, Vellend provides a general and unificatory theory. The target variables of lower-level laws should be translated into 
these higher-level forces to create a unified science. All ecological communities have their composition determined by different degrees of these forces. This theory is both economical, and hopefully explanatorily complete, avoiding the pitfalls of having numerous laws without a clear sense of their relationship.

Vellend explicitly describes his theory as an attempt to unify local community phenomena under a set of higher-level processes. To show how this theory unifies community ecology under higher-level processes Vellend utilizes Elliot Sober's (1991; 2000) discussion of natural selection. Sober argues that natural selection buys its generality through abstracting away all the causes of fitness differences. These causes can be explained by lower-level laws. Instead, it is a theory of the consequences of fitness differences. This act of abstraction unites the different causes that contribute to fitness and provides an explanatory schematic that applies across biology. Vellend aims to do likewise, focusing on the distribution and abundance of organism types that are the product of ecological processes.

This act of abstraction removes the messiness Vellend, and I, contends exists in ecological theory. He argues current ecological theory is a laundry list of patterns, lowerlevel processes, higher-level processes, and the numerous different ecological kinds. By creating a general explanatory schema, built around higher-level processes, he aims to unite the many lower-level generalizations into a single framework. This provides an explanatory schema and structures the observations and inferences we make about 
communities. The invariant generalizations described by both Colyvan and Ginzburg (2003) and Linquist et al. (2016) can be described within this framework as lower-level processes, which contribute to the higher-level processes described by Vellend (2016). For example, the lower-level law, "the impact of grazers on prey biomass decreases in proportion to species richness of prey communities," is incorporated into the higherlevel processes of ecological selection (Linquist et al. 2016).

Describing selection as just one of the four processes involved in community ecology laws helps explain the problems with Lawton's (1999) view of community laws. He was just too narrow in what should count as a law in community ecology, relegating too much variation to background conditions. When Lawton describes community ecology, he only counts local populations and their interactions as being in the scope of community ecology, not metacommunity dynamics such as migration between communities, which are treated as background conditions. One way to interpret the failure of local community ecology is there are few robust local relationships given variation in the metacommunity dynamics. All these other factors are included within the Vellend framework.

Now I am not defending the 'theory of community ecology' as being correct. There will be difficulty implementing these four axes. Other grand theories like Thomas Schoener's (1986), which had 12 axes in a $n$-dimensional hyperspace to explain community assemblage, could not be implemented due the complexity and contingency 
of the different axes (Lawton 1999). Some believe ecology's causal heterogeneity means that general laws cannot ever be found (Elliott-Graves 2018).

Another worry is that the expanandum of 'the science' is not worthy of general unifying theories. The domain of a unifying explanation is partially derived from the epistemic aims of a science. As I described earlier a unifying explanation for community ecology would have explanatory power for the entirety of an ecological community. There could be the possibility that explanation in community ecology is a problem because ecological communities do not exist (Ricklefs 2008; Shrader-Frechette and McCoy 1994, 110). If true one could interpret the fracturing of the science into piecemeal explanation which don't easily relate is the explication of the science into more apt sciences as they have better defined target systems. This style of argument has some force which needs to be mitigated and I have defended the explanatory value of ecological communities previously (Lean 2018).

But such issues, among others, do not diminish the interesting contribution to ecological theory, and laws of nature, presented by Vellend (2016). He provides a comprehensive and explicit attempt to unify lower-level ecological laws into a general higher-level explanatory framework, which deserves further consideration.

\section{CONCLUSION}


The science of community ecology lacks explanatory integration. The variables and laws described in the many different local laws do not easily integrate with the other local laws. This is because ecological variables are inconsistent and incommensurable. Without a higher-level theory to organize the lower-level explanations we will struggle to provide comprehensive explanations of ecological phenomena. Some ecologists, particularly Mark Vellend, realize this and are actively attempting to address these issues by presenting a general theory of ecological communities (Vellend 2016). Philosophers should pay attention to these developments as they provide insight into scientific explanation and the historical debates around laws in ecology.

\section{ACKNOWLEDGMENTS}

First I would like to thank my reviewers for the helpful feedback. Particular thanks goes to Stefan Linquist, who provided enthusiastic and insightful criticism. Cheers to Mark Colyvan, Ross Pain, and Matt Spike as well. The views expressed in this paper do not reflect on theirs. Work on this paper was supported by an Australian Research Council Discovery Grant (grant number DP170104924).

\section{REFERENCES}


Batterman, R. W., and C. C. Rice. 2014. "Minimal Model Explanations." Philosophy of Science 81(3): 349-76.

Bradshaw, C. J. A. 2012, September 17. "Ecology Is a Tower of Babel." Retrieved August 2, 2019, from ConservationBytes.com website: https://conservationbytes.com/2012/09/17/ecology-tower-babel/.

Brown, J. H. 1995. Macroecology. Chicago: University of Chicago Press.

Cartwright, N. 1983. How the Laws of Physics Lie. Oxford: Oxford University Press.

Chase, J. M., and M. A. Leibold. 2003. Ecological Niches: Linking Classical and Contemporary Approaches. Chicago: University of Chicago Press.

Colyvan, M., and L. R. Ginzburg. 2003. "Laws of Nature and Laws of Ecology." Oikos 101(3): 649-53.

Cooper, G. 1998. "Generalizations in Ecology: A Philosophical Taxonomy." Biology and Philosophy 13(4): 555-86.

Craver, C. F. 2007. Explaining the Brain: Mechanisms and the Mosaic Unity of Neuroscience. Oxford: Oxford University Press.

Devictor, V., D. Mouillot, C. Meynard, F. Jiguet, W. Thuiller, and N. Mouquet. 2010. "Spatial Mismatch and Congruence between Taxonomic, Phylogenetic and Functional Diversity: The Need for Integrative Conservation Strategies in a Changing World." Ecology Letters 13(8): 1030-40. 
Elliott-Graves, A. 2018. "Generality and Causal Interdependence in Ecology." Philosophy of Science 85(5): 1102-14.

Elton, C. S. 1927. Animal Ecology. Chicago: University of Chicago Press.

Ginzburg, L., and M. Colyvan. 2004. Ecological Orbits: How Planets Move and Populations Grow. Oxford: Oxford University Press.

Grimm, V., and C. Wissel. 1997. "Babel, or the Ecological Stability Discussions: An Inventory and Analysis of Terminology and a Guide for Avoiding Confusion." Oecologia 109(3): 323-34.

Herrando-Pérez, S., S. Delean, B. W. Brook, and C. J. A. Bradshaw. 2012. "Density Dependence: An Ecological Tower of Babel." Oecologia 170(3): 585-603.

Hutchinson, G. E. 1957. "Concluding Remarks." Population Studies: Animal Ecology and Demography. Cold Springs Harbor Symposia on Quantitative Biology (22): 41527.

Inkpen, S. A., G. M. Douglas, T. D. P. Brunet, K. Leuschen, W. F. Doolittle, and M. G. Langille. 2017. "The Coupling of Taxonomy and Function in Microbiomes." Biology \& Philosophy 32(6): 1225-43.

Kingsland, S. E. 1985. Modeling Nature. Chicago: University of Chicago Press.

Kitcher, P. 1981. "Explanatory Unification." Philosophy of Science 48(4): 507-31.

Lange, M. 2005. "Ecological Laws: What Would They Be and Why Would They Matter?" Oikos 110(2): 394-403. 
Lawton, J. H. 1999. "Are There General Laws in Ecology?" Oikos 84(2): 177-192.

Lean, C. H. 2017. "Biodiversity Realism: Preserving the Tree of Life." Biology \& Philosophy 32(6): 1083-1103.

Lean, C. H. 2018. "Indexically Structured Ecological Communities." Philosophy of Science 85(3): 501-22.

Linquist, S. 2015. "Against Lawton's Contingency Thesis; or, Why the Reported Demise of Community Ecology Is Greatly Exaggerated." Philosophy of Science 82(5): 110415.

Linquist, S., T. R. Gregory, T. A. Elliott, B. Saylor, S. C. Kremer, and K. Cottenie. 2016. "Yes! There Are Resilient Generalizations (or 'Laws') in Ecology." Quarterly Review of Biology 91(2): 119-31.

MacArthur, R. H. 1958. "Population Ecology of Some Warblers of Northeastern Coniferous Forests." Ecology39(4): 599-619.

MacArthur, R., and R. Levins. 1967. "The Limiting Similarity, Convergence, and Divergence of Coexisting Species." American Naturalist 101(921): 377-85.

Mazel, F., M. W. Pennell, M. W. Cadotte, S. Diaz, G. V. Dalla Riva, R. Grenyer, C. M. Tucker, et al. 2018. "Prioritizing Phylogenetic Diversity Captures Functional Diversity Unreliably." Nature Communications 9(1): 2888.

Mitchell, S. D. 2000. "Dimensions of Scientific Law." Philosophy of Science 67(2): 242-65. 
Mlambo, M. C. 2014. "Not All Traits Are 'Functional': Insights from Taxonomy and Biodiversity-Ecosystem Functioning Research." Biodiversity and Conservation 23(3): 781-90.

Peters, R. H. 1991. A Critique for Ecology. Cambridge: Cambridge University Press.

Rejmánek, M., D. M. Richardson, M. G. Barbour, M. J. Crawley, G. F. Hrusa, P. B. Moyle, M. Williamson, et al. 2002. "Biological Invasions: Politics and the Discontinuity of Ecological Terminology." Bulletin of the Ecological Society of America 83(2): 13133.

Ricklefs, R. E. 1987. “Community Diversity: Relative Roles of Local and Regional Processes." Science 235(4785): 167-71.

Ricklefs, R. E. 2008. "Disintegration of the Ecological Community." American Naturalist 172(6): 741-50.

Roughgarden, J. 2009. "Is There a General Theory of Community Ecology?" Biology \& Philosophy 24(4): 521-29.

Roughgarden, J. 1989. "The Structure and Assembly of Communities." In Perspectives in Ecological Theory, edited by Roughgarden J, May RM, Levin SA, pp 203-226. Princeton: Princeton University Press

Sagoff, M. 2005. "Do Non-native Species Threaten the Natural Environment?" Journal of Agricultural and Environmental Ethics 18(3): 215-36. 
Schoener, T. W. 1986. "Overview: Kinds of Ecological Communities: Ecology Becomes Pluralistic." In Community Ecology, edited by J. M. Diamond and T. J. Case, pp. 467-79. New York: Harper and Row.

Shrader-Frechette, K. S., and E. D. McCoy. 1990. "Theory Reduction and Explanation in Ecology." Oikos 58(1): 109-114.

Shrader-Frechette, K. S., and E. D. McCoy. 1993. Method in Ecology: Strategies for Conservation. Cambridge: Cambridge University Press.

Shrader-Frechette, K. S., and E. D. McCoy. 1994. "How the Tail Wags the Dog: How Value Judgments Determine Ecological Science." Environmental Values 3(2): 107-20.

Skyrms, B. 1980. Causal Necessity: A Pragmatic Investigation of the Necessity of Laws. New Haven: Yale University Press.

Smart, J. J. C. 1959. "Can Biology Be an Exact Science?" Synthese 11(4): 359-68.

Smart, J. J. C. 1963. Philosophy and Scientific Realism. London: Routledge.

Sober, E. 2000. Philosophy of Biology (2nd ed., vol. 85). Boulder, CO: Westview Press.

Sober, E. 1991. "Models of Cultural Evolution." In Trees of Life: Essays in the Philosophy of Biology, edited by P. E. Griffiths, pp. 17-38. New York: Kluwer.

Trombley, C. A., and K. Cottenie. 2019. "Quantifying the Scientific Cost of Ambiguous Terminology in Community Ecology." Philosophical Topics (this volume).

Turchin, P. 2001. "Does Population Ecology Have General Laws?" Oikos 94(1): 17-26. 
Vellend, M. 2010. "Conceptual Synthesis in Community Ecology." Quarterly Review of Biology 85(2): 183-206.

Vellend, M. 2016. The Theory of Ecological Communities (Vol. 75). Princeton, NJ: Princeton University Press.

Vellend, M., and J. L. Orrock. 2009. "Ecological and Genetic Models of Diversity: Lessons across Disciplines." In The Theory of Island Biogeography Revisited, edited by J. B. Losos and R. E. Ricklefs, pp. 439-61. Princeton: Princeton University Press.

Wayne, A. 2018. "Explanatory Integration." European Journal for Philosophy of Science 8(3): 347-65.

Weisberg, M. 2013. Simulation and Similarity: Using Models to Understand the World. Oxford: Oxford University Press.

Winter, M., V. Devictor, and O. Schweiger. 2013. "Phylogenetic Diversity and Nature Conservation: Where Are We?" Trends in Ecology \& Evolution 28(4): 199-204.

Woodward, J. 2005. Making Things Happen: A Theory of Causal Explanation. Oxford: Oxford University Press.

Woodward, J. 2017. "Scientific Explanation." In E. N. Zalta (Ed.), The Stanford Encyclopedia of Philosophy (Fall 2017). Retrieved on December 2, 2019, from https://plato.stanford.edu/archives/fall2017/entries/scientific-explanation/.

Woodward, J. 1997. "Explanation, Invariance, and Intervention." Philosophy of Science 64:S26-S41. 